\title{
Contribuição da tomografia helicoidal no estadiamento pré-operatório do carcinoma gástrico
}

\author{
Contribution of abdominal helical tomography in the preoperative staging of gastric carcinoma
}

Flávio Mendes de Oliveira', Nadia Pavarini², Juliana Ortiz², Aline Biral Zanon², Lourdes Conceição Martins³, Wilson Roberto Catapani ${ }^{4}$, Ana Maria Amaral Antonio Mader ${ }^{5}$, Jaques Waisberg ${ }^{6}$

\begin{abstract}
RESUMO
Objetivo: Analisar a sensibilidade da tomografia helicoidal abdominal na detecção do comprometimento linfonodal e de metástases hepáticas e peritoneais no estadiamento préoperatório do carcinoma gástrico. Método: Foram estudados retrospectivamente 52 doentes operados por carcinoma gástrico e estadiados por tomografia helicoidal abdominal com contraste oral e intravenoso. 0s resultados baseados nos laudos correspondentes foram correlacionados com os achados intraoperatórios e exame anatomopatológico dos espécimes cirúrgicos. Resultados: A neoplasia gástrica localizou-se na região do antropilórica em 38 (73,1\%) doentes, na cárdia em 8 (15,4\%) e no corpo e fundo em 6 (11,5\%). A tomografia helicoidal abdominal detectou lesão gástrica em 25 (48,1\%) doentes, metástases hepáticas em $6(11,5 \%)$, acometimento de linfonodos em $5(9,6 \%)$, ascite em 4 $(7,7 \%)$ e comprometimento no fígado, baço e pâncreas em $1(1,9 \%)$. Na laparotomia, constataram-se metástases hepáticas em $6(11,5 \%)$ doentes e ascite em 12 (23,1\%). Nos doentes submetidos à ressecção com intenção curativa da neoplasia, foram identificados pela tomografia helicoidal abdominal pré-operatória 27 (67,5\%) linfonodos sugestivos de comprometimento. 0 exame anatomopatológico mostrou $16(40,0 \%)$ doentes com linfonodos infiltrados pelo carcinoma gástrico. A sensibilidade do exame de tomografia helicoidal abdominal na identificação pré-operatória da lesão gástrica foi 48,1\%, para linfonodos acometidos foi de 18,5\% e para metástases hepáticas a sensibilidade foi 100,0\%. Treze (25\%) doentes apresentaram metástases peritoneais não reveladas pela tomografia helicoidal. Conclusões: 0 exame de tomografia computadorizada helicoidal abdominal no pré-operatório do carcinoma gástrico apresentou sensibilidade elevada na detecção de metástases hepáticas, sensibilidade baixa na deteç̧ão do comprometimento linfonodal pela neoplasia e não foi capaz de identificar metástases peritoneais.
\end{abstract}

Palavras-chave: Neoplasias gástricas; estadiamento de neoplasias; tomografia computadorizada espiral; hiperplasia do linfonodo gigante; metástase neoplásica.

\section{ABSTRACT}

Objective: To analyze the sensitivity of preoperative abdominal helical tomography of gastric carcinoma in detecting lymph node compromise and hepatic and peritoneal metastases. Method: We examined retrospectively 52 patients with gastric carcinoma who had undergone surgery. Patients were staged in according to the findings using abdominal helical tomography with oral and intravenous contrast. Results of pre-operative abdominal tomography correlated with intra-operative findings and anatomopathological results. Results: Gastric neoplasia was located in the antropyloric region in 38 patients (73.1\%), in the cardia region in 8 (15.4\%), and in the body and fundus in $6(11.5 \%)$. Abdominal tomography detected gastric lesions in 25 patients $(48.1 \%)$, revealed hepatic metastases in $6(11.5 \%)$, lymph node invasion in $5(9.6 \%)$, ascitis in $4(7.7 \%)$, and neoplastic infiltration in the liver, spleen, and pancreas in $1(1.9 \%)$. Laparotomy detected hepatic metastases in $6(11.5 \%)$ patients and ascitis in $12(23.1 \%)$. Among the patients submitted to curative resection, 27 (67.5\%) were identified as having lymph nodes compromised on preoperative abdominal tomography. Anatomopathological examination of the operative specimen showed that 16 patients (40.0\%) had lymph nodes invaded by carcinoma. The sensitivity of pre-operative abdominal tomography exams in identifying gastric lesion was $48.1,18.5 \%$ for invaded lymph nodes, and $100.0 \%$ for hepatic metastases. Although 13 (25\%) patients presented peritoneal metastases intra-operatively, the abdominal tomography exams did not reveal such lesions. Conclusions: Exams of the gastric carcinoma presented high sensitivity in detecting hepatic metastases, low sensitivity in detecting lymph node invasion by neoplasia, and inability to identify peritoneal metastases.

Keywords: Stomach neoplasms; neoplasm staging; tomography, spiral computed; giant lymph node hyperplasia; neoplasm metastasis.

\section{Recebido: 8/6/2009}

Revisado: $11 / 8 / 2010$

Aprovado: 23/8/2010

Trabalho realizado no Hospital Estadual "Mário Covas" da Faculdade de Medicina do ABC (FMABC), Santo André (SP), Brasil

1 Departamento de Morfologia e Fisiologia da Faculdade de Medicina do ABC (FMABC), Santo André, (SP) Brasil

2 Departamento de Saúde Materno-Infantil da Faculdade de Medicina do ABC (FMABC), Santo André, (SP) Brasil

${ }^{3}$ Disciplina de Saúde Coletiva do Departamento de Saúde da Coletividade da Faculdade de Medicina do ABC (FMABC), Santo André, (SP) Brasil

${ }^{4}$ Disciplina de Gastroenterologia do Departamento de Clínica Médica da Faculdade de Medicina do ABC (FMABC), Santo André, (SP) Brasil

${ }^{5}$ Disciplina de Patologia Geral do Departamento de Patologia da Faculdade de Medicina do ABC (FMABC), Santo André, (SP) Brasil

${ }^{6}$ Disciplina de Cirurgia do Aparelho Digestivo do Departamento de Cirurgia da Faculdade de Medicina do ABC (FMABC), Santo André, (SP) Brasil

Endereço para correspondência: Jaques Waisberg - Rua das Figueiras, 550, apto. 134 - Bairro Jardim - CEP 09080-300 - Santo André (SP), Brasil - Tel.: (11)

4436-2461 - Fax: (11) 4436-8739 - e-mail: jaqueswaisberg@uol.com.br 


\section{Introdução}

Cerca de 10 a 30\% dos doentes com carcinoma gástrico são inoperáveis no momento do diagnóstico pela presença de metástases. Metade do restante deles apresenta lesão irressecável durante a laparotomia ${ }^{1-3}$.

No Brasil, o carcinoma gástrico representa a principal causa de morte entre todas as doenças malignas do aparelho digestivo ${ }^{4}$. É o primeiro em frequência no homem e o terceiro na mulher, depois de mama e colo de útero ${ }^{1}$. O número de casos novos estimados para o Brasil no ano de 2008 é de 14.080 entre homens e de 7.720 nas mulheres 5 . Estes valores correspondem ao risco estimado de 15 casos novos a cada 100 mil homens e 8 para cada 100 mil mulheres ${ }^{5}$. O pico de prevalência da doença ocorre entre 50 e 70 anos de idade ${ }^{1}$. A maioria dos casos de câncer gástrico no Brasil também se apresenta como doença avançada, dos quais $60 \%$ se associam à metástase linfonodal. A forma precoce é encontrada em menos de $16 \%$ dos pacientes com essa afecção 5 .

Os objetivos do estadiamento do carcinoma gástrico são detectar metástases à distância e determinar o risco de recidiva avaliado pela invasão tumoral e acometimento de linfonodos ${ }^{6}$. Desse modo, o estadiamento pré-operatório identifica os doentes que vão se submeter à operação curativa e aqueles que não têm esperança de cura ou não vão ter uma melhoria de qualidade de vida com a ressecção da neoplasia ${ }^{7}$.

A tomografia computadorizada (TC) tem sido utilizada para detecção ${ }^{8}$, estadiamento e seguimento de neoplasias gastrintestinais?. Por possuir algumas vantagens sobre outros métodos diagnósticos convencionais, sua tecnologia vem sendo cada vez mais utilizada e desenvolvida ${ }^{10}$.

Imagens axiais de TC têm sido utilizadas para o estadiamento de neoplasias gástricas e é considerada a modalidade de estadiamento de escolha porque pode auxiliar a identificar o tumor primário, avaliar a disseminação local e detectar o comprometimento linfonodal e metástases à distância ${ }^{11}$.

$\mathrm{Na}$ TC helicoidal multislice, uma grande quantidade de detectores de raios X é utilizada para obter até 16 digitalizações espirais simultaneamente ${ }^{11}$. Com essa técnica, é possível "fotografar" todo o tórax e abdome superior em inspiração. Consequentemente, artefatos de movimento causados pela respiração ou peristalse são bastante reduzidos. Além disso, os dados podem ser reconstruídos com resolução espacial quase isotrópica, permitindo alta qualidade multiplanar ou reconstrução no padrão tridimensional (3D) ${ }^{3,11}$. Técnicas adequadas de injeção de contraste oferecem melhor diferenciação do tecido neoplásico da mucosa normal ${ }^{3,5,11}$. A utilização da TC helicoidal multislice pode elevar o índice de detecção da neoplasia.

A detecção pré-operatória de linfonodos alterados ainda é um desafio para qualquer modalidade diagnóstica, especialmente de linfonodos com tamanho normal ${ }^{2}$. Na suspeita de comprometimento linfonodal, o cirurgião deve procurar esta área durante a operação. Isso pode modificar a conduta cirúrgica tanto por alargar as margens da ressecção cirúrgica como por determinar que a lesão é irressecável³.
A precisão da TC em determinar o aumento do grau de invasão do tumor quando comparada ao estadiamento TNM é controversa. Somado à dificuldade na identificação de carcinomatose peritoneal, o desempenho da TC pode resultar em subestadiamento ${ }^{6,12,13}$.

Doentes com câncer gástrico são submetidos à laparotomia quando a avaliação pré-operatória falha em demonstrar doença metastáti$\mathrm{ca}^{13}$. Entretanto, a maioria desses doentes apresenta metástases para o peritônio ou fígado que não são diagnosticadas até a operação ${ }^{3}$. A laparotomia é pouco benéfica nesses doentes porque eles possuem média de sobrevivência de apenas três a nove meses ${ }^{3,6,14}$.

O objetivo do presente estudo foi analisar a sensibilidade do exame de TC helicoidal abdominal na deteç̧ão do comprometimento linfonodal, metástases hepáticas e metástases peritoneais no pré-operatório de doentes com carcinoma gástrico.

\section{Método}

Trata-se de estudo descritivo, analítico e retrospectivo. Foi realizado levantamento dos prontuários de 52 doentes diagnosticados com carcinoma gástrico (CAG) no Hospital Estadual "Mario Covas" (Santo André, São Paulo) no período de novembro de 2002 a dezembro de 2006.

O projeto de pesquisa foi aprovado pelo Comitê de Ética em Pesquisa (CEP) da Faculdade de Medicina da Fundação do ABC (parecer número 055/2007). Este estudo foi realizado de acordo com os padrões éticos aceitos pela declaração de Helsinki da Associação Médica Mundial, adotado em 1964 e emendado em 1996.

Foram incluídos 52 doentes neste estudo, sendo 34 homens $(65,4 \%)$ e 18 mulheres $(34,6 \%)$ com média de idade de $62,5 \pm 14,3$ anos (29 a 87 anos).

As informações clínicas e morfológicas foram obtidas por consultas em prontuários hospitalares ou por entrevista com os doentes ou familiares nos retornos ambulatoriais.

O estadiamento pré-operatório dos doentes do presente estudo foi realizado pelo exame físico, radiografia torácica, ultrassonografia abdominal e tomografia helicoidal do abdome.

No caso da realização de ressecções curativas ou paliativas, o produto da gastrectomia foi encaminhado para o exame anatomopatológico. Nos doentes submetidos a operações paliativas sem ressecção gástrica, os dados anatomopatológicos foram fornecidos a partir das biópsias endoscópicas pré-operatórias e realizadas durante a operação. As operações foram conduzidas pela mesma equipe cirúrgica.

Foi realizada análise dos laudos respectivos dos exames de TC helicoidal abdominal pré-operatória dos doentes incluídos no estudo. Dois radiologistas avaliaram os exames, um como relator e outro como revisor. Em caso de avaliação discordante, os exames eram discutidos por eles e apenas um laudo consensual era emitido.

Os achados tomográficos foram comparados com os achados intraoperatórios e com os aspectos morfológicos da neoplasia, verifi- 
cando-se a acurácia da TC helicoidal abdominal no estadiamento préoperatório do carcinoma gástrico. O aparelho de tomografia helicoidal utilizado neste estudo foi modelo High Speed (General Electric Company, Estados Unidos). Na técnica de realização dos exames tomográficos foram efetuados cortes axiais com espessura de $10 \mathrm{~mm}$ e $10 \mathrm{~mm}$ de intervalo na fase pré-contraste, abrangendo apenas o abdome superior. $\mathrm{Na}$ fase pós-contraste imediata, foram realizados cortes com espessura de $7 \mathrm{~mm}$ e intervalo de $8 \mathrm{~mm}$ no abdome superior e na fase tardia foram realizados cortes com $7 \mathrm{~mm}$ de espessura e $8 \mathrm{~mm}$ de intervalo no abdome superior e $5 \mathrm{~mm}$ de espessura por $7 \mathrm{~mm}$ de intervalo na pelve.

Foi ministrado previamente ao exame tomográfico contraste iodado (ioxitalamato de meglubina, Telebrix ${ }^{\circledR}$, Guerbet do Brasil, São Paulo) diluído em água na quantidade de $30 \mathrm{~mL}$ em 1,5 litros de água. Três horas antes da realização do exame, iniciou-se a ministração do contraste via oral, sendo que os últimos $150 \mathrm{~mL}$ desta diluição foram dados imediatamente antes do início do exame. Após a fase sem contraste intravenoso, foram ministrados $100 \mathrm{~mL}$ de contraste intravenosos (ioxitalamato de meglubina, Telebrix ${ }^{\circledR}$, Guerbet do Brasil, São Paulo).

Os critérios utilizados na interpretação tomográfica do estadiamento do carcinoma gástrico foram os seguintes: i. espessura parietal gástrica - espessamento parietal gástrico focal ou difuso com realce após injeção do meio de contraste iodado superior a $20 \mathrm{~mm}$, considerando-se preparo adequado, com boa distensão hídrica da luz gástrica para permitir a noção exata da extensão, tanto em superfície quanto em profundidade; ii. exploração das cadeias linfonodais: perivisceral e à distância (hilo hepático, peripancreático, retroperitoneal, espaço inframediastinal posterior e mediastino); iii. pesquisa de metástase: por continuidade com órgãos vizinhos, visceral (hepática, ovariana) e peritoneal. Para a pesquisa de linfonodos foi utilizado o corte de $8 \mathrm{~mm}$ de espessura. Os linfonodos foram estadiados como N1 (1 a 6 nódulos), N2 (7 a 15 nódulos) e N3 (mais de 15 nódulos), de acordo com o número de linfonods metastáticos regionais encontrados.

Neste estudo, foram considerados critérios de inclusão a presença de carcinoma gástrico confirmado pelo estudo histopatológico da biópsia da lesão obtida por gastroscopia, a realização de operação com intenção curativa ou paliativa, a realização pré-operatória de TC helicoidal abdominal completa e tecnicamente satisfatória, incluindo ministração de contraste via oral e intravenosa e obtenção de laudo de exame anatomopatológico de biópsias e/ou peça de ressecção gástrica e de demais estruturas. Foram considerados critérios de exclusão a presença de outras neoplasias gástricas além de carcinomas, ausência de exame anatomopatológico confirmatório de CAG e prontuário com informações incompletas ou faltantes para o preenchimento integral do protocolo do estudo.

No tocante às variáveis biodemográficas foram analisadas idade e sexo. Em relação ao carcinoma gástrico foram estudados os seguintes aspectos: localização da lesão gástrica no exame de endoscopia digestiva alta pré-operatória; achados da tomografia helicoidal abdominal pré-operatória (localização da lesão no estômago, presença de com- prometimento linfonodal e à distância, presença de ascite); achados intraoperatórios (localização da neoplasia gástrica, presença de carcinomatosis e/ou ascite, extensão da neoplasia no maior eixo); tipo de procedimento cirúrgico realizado; achados do exame anatomopatológico da neoplasia gástrica (número de linfonodos acometidos pela neoplasia nos doentes submetidos à ressecção com intenção curativa da lesão, presença de células em anel de sinete, grau de diferenciação celular e estadiamento inicial (Ia, Ib ou II) ou avançado (IIIa, IIIb ou IV) baseado na classificação TNM da UICC.

Todos os laudos anatomopatológicos foram feitos pela mesma patologista (AMAAM), de acordo com o padrão de análise macroscópica e microscópica de neoplasias gástricas adotado pelo Serviço de Anatomia Patológica do Hospital Estadual Mário Covas.

$\mathrm{Na}$ avaliação dos resultados, as variáveis qualitativas foram representadas por frequência absoluta $(\mathrm{N})$ e relativa (porcentagem). Foram utilizados como modelos estatísticos a média aritmética e desvio padrão, e o teste de sensibilidade. Em todos os testes, fixou-se em 0,05 ou $5 \%$ o nível para a rejeição da hipótese de nulidade (intervalo de confiança de $95 \%$ ) de acordo com os padrões correntes em estudos biológicos, assinalando-se com asterisco os valores significativos.

\section{Resultados}

De acordo com os resultados da endoscopia digestiva alta realizada em todos os 52 doentes, a neoplasia gástrica estava localizada na região do antropilórica em 38 (73,1\%) doentes, na região da cárdia em $8(15,4 \%)$ e na região do corpo e fundo em $6(11,5 \%)$.

No procedimento operatório, 46 doentes apresentaram lesão gástrica nas seguintes localizações: 16 no antro, 7 na região antropilórica 4 no corpo, 4 em todo estômago, 3 na cárdia, 3 no corpo e no antro, 1 na transição esofagogástrica, 1 na cárdia e no fundo, 1 no fundo e 1 no piloro. Em 6 (11,5\%) casos não havia indicação do local da lesão na descrição da operação. Em 5 (9,6\%) enfermos observou-se carcinomatose peritoneal. Vinte e quatro $(46,2 \%)$ enfermos possuíam neoplasia com extensão no maior eixo $\leq 5 \mathrm{~cm}$ e $28(53,8 \%)$ apresentaram lesão $>5 \mathrm{~cm}$.

Dentre os 40 (76,9\%) doentes operados com intenção curativa, foi realizada gastrectomia total em $21(40,4 \%)$ e gastrectomia parcial em 19 (36,5\%). As operações paliativas realizadas foram gastroenteroanastomose em 7 (13,5\%) enfermos, jejunostomia em 4 (7,7\%) e laparoscopia diagnóstica em 1 (1,9\%). Três gastrectomias totais foram ampliadas com esofagectomia e três com pancreatectomia córporo-caudal e esplenectomia. Doze (23,08\%) enfermos apresentavam lesões irressecáveis e $13(25,0 \%)$ apresentavam carcinomatose peritoneal.

Todos os 52 doentes apresentaram carcinoma gástrico no exame de anatomopatológico. Dentre esses doentes, o exame anatomopatológico apontou que 19 (36,5\%) apresentavam comprometimento neoplásico linfonodal e em 28 (53,8\%) enfermos, os linfonodos estavam livres de neoplasia (Figura 1). Vinte e um (40,38\%) doentes apresentaram carcinoma gástrico com células em anel de sinete. Quanto ao 
grau de diferenciação, 25 (48,1\%) lesões eram de alto grau, 24 (46,1\%) de baixo grau e em $3(5,8 \%)$ laudos do exame anatomopatológico não constava essa informação. Dos doentes desta série, 19 (36,5\%) foram classificados como estádio inicial (Ia, Ib ou II) e 33 (63,5\%) como estádio avançado (IIIa, IIIb ou IV) da classificação TNM da UICC para o carcinoma gástrico.

No estudo tomográfico dos 52 doentes desta série, a TC helicoidal abdominal detectou lesão gástrica em 25 (48,1\%), sendo descritos 19 exames com espessamento de parede, três com lesão neoplásica gástrica, um espessamento de parede sugestiva de lesão neoplásica, um tumor aderido ao lobo esquerdo do fígado e um com estenose antropilórica. Em 27 (51,9\%) doentes, a tomografia helicoidal não detectou lesão parietal gástrica.

No tocante ao comprometimento hepático, a TC helicoidal abdominal detectou metástases hepáticas em $7(13,4 \%)$ doentes, todas confirmadas durante a operação. Em 45 (86,5\%) doentes não se encontrou referência às metástases hepáticas no laudo do exame, assumindo-se, então, que não foram detectadas (Figura 2). Dentre estes doentes, outros $4(7,7 \%)$ apresentavam metástases hepáticas constatadas no intraoperatório. Todos os casos de metástases hepáticas verificadas na operação foram confirmados pelo exame histopatológico de material de biópsia intraoperatória.

A presença de linfonodos comprometidos foi descrita na tomografia helicoidal em $5(9,6 \%)$ doentes, sendo confirmados pelo laudo do exame anatomopatológico em 4 (7,7\%) deles. Por outro lado, em 47 (90,3\%) doentes não havia descrição do aumento de linfonodos perigástricos ou regionais na TC helicoidal abdominal, assumindo-se, então, que não houve detecção de linfadenomegalia.

Dentre os doentes submetidos à ressecção com intenção curativa da neoplasia, foram identificados 27 (67,5\%) com linfonodos sugestivos de comprometimento pela neoplasia. O exame anatomopatológico da peça operatória mostrou que em $16(40,0 \%)$ desses doentes havia linfonodos infiltrados pelo carcinoma gástrico.

A sensibilidade do exame de TC helicoidal abdominal com contraste oral e intravenoso no pré-operatório de doentes com carcinoma gástrico na identificação da neoplasia no estômago foi de 48,1\%, para linfonodos acometidos foi de 18,5\%, e para metástases hepáticas foi de 100,0\%. Embora 13 (25\%) doentes apresentassem metástases peritoneais constatadas pelo achado intraoperatório e confirmadas pelo resultado do exame anatomopatológico, a presença dessas lesões não foi constatada por esse exame e, consequentemente, sua sensibilidade foi considerada nula.

\section{Discussão}

A estimativa intraoperatória da infiltração do tumor e de metástases linfonodais são aspectos fundamentais para a decisão sobre qual operação deve ser realizada no doente com CAG. A controvérsia da acurácia do estadiamento do CAG pela TC é, em parte, alimentada pelo

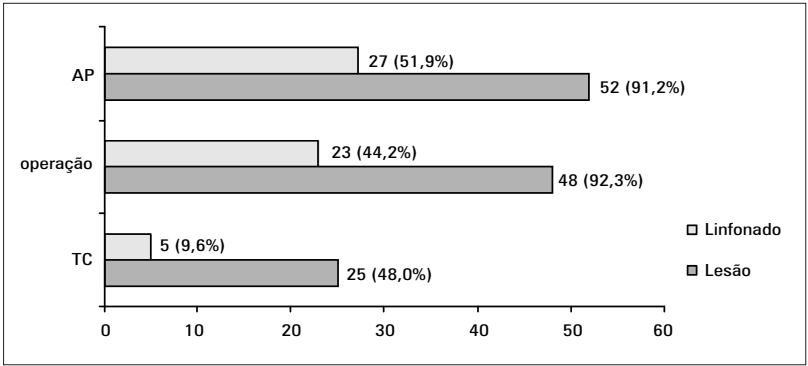

Figura 1 - Gráfico comparativo dos resultados positivos da presença de carcinoma e acometimento de linfonodos entre a tomografia helicoidal abdominal (TC), achados intraoperatórios e exame anatomopatológico (AP) nos doentes com carcinoma gástrico.

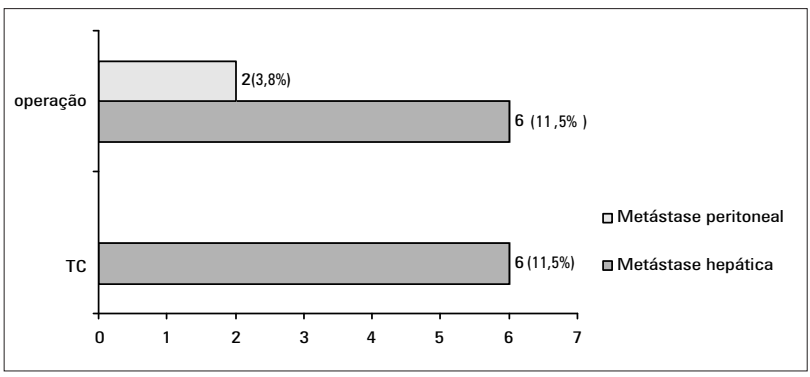

Figura 2 - Gráfico comparativo da detecção de metástases hepáticas e peritoneais entre a tomografia helicoidal abdominal (TC) e a operação realizada nos doentes com carcinoma gástrico.

fato de que os equipamentos e técnicas utilizadas nos diversos estudos variam consideravelmente $e^{15}$. As diferenças incluem a variação do tempo de escaneamento e a variação no uso de material de contraste oral e venoso?. A maioria dos estudos utiliza o critério de tamanho de 8-15 $\mathrm{mm}$ para os linfonodos não comprometidos ${ }^{2,4,16-21}$.

No entanto, existe falha diagnóstica nesta avaliação em cerca de $50 \%$ dos $\operatorname{casos}^{22}$. Habitualmente, o estadiamento da profundidade de penetração da neoplasia nas camadas da parede gástrica ( $\mathrm{T}$ ) é subestimado e o comprometimento linfonodal (N) é hiperestimado ${ }^{22}$. Portanto, como descrito por Ziegler et al. ${ }^{8}$, apenas o estadiamento intraoperatório não permite a seleção do melhor procedimento cirúrgico para o doente. Como habitualmente não são aplicados critérios TNM para o exame de tomografia, esta seleção fica principalmente dependente da avaliação intraoperatória ${ }^{21-24}$. Procedimentos cirúrgicos com exame de congelação de linfonodos suspeitos de comprometimento neoplásico e de margens de ressecção devem tornar mais acurados o estadiamento $\mathrm{T}$ e o $\mathrm{N}$ do $\mathrm{CAG}^{8}$.

Quanto aos linfonodos, a detecção pré-operatória daqueles acometidos pela neoplasia constitui desafio para qualquer modalidade diagnóstica, especialmente quando se consideram aqueles de tamanho normal $^{2,25}$. Provavelmente, isto acontece pelo elevado valor de corte (cut-off) de $10 \mathrm{~mm}$ entre o linfonodo considerado normal e o considerado aumentado de tamanho, cuja sensibilidade varia entre 22 e $73 \%$ 2,10. Com o estômago não distendido, a delineação dos linfonodos e da parede gástrica podem ser melhorados, já que linfonodos maiores podem 
não ser visibilizados se estiverem próximos à lesão ${ }^{18}$. A sensibilidade de detecção de linfonodos aumentados da presente casuística foi menor do que a observada na literatura. Isso foi atribuído à espessura do corte utilizado de $7 \mathrm{~mm}$, valor considerado maior do que o utilizado habitualmente na TC helicoidal nos estudos de estadiamento do CAG.

No estudo de Sussman et al. ${ }^{20}$, a TC revelou sensibilidade de $67 \%$ para a detecção de linfadenopatia e foi considerada pouco expressiva. Embora outras publicações ${ }^{4,10,11}$ tenham apontado sensibilidade de até $97 \%$, estudo prospectivo ${ }^{1}$ relatou sensibilidade da TC para a detecção de linfadenopatia maligna de $43 \%$ quando comparada com o achado cirúrgico. A TC apresenta dificuldade para a detecção de linfonodos adjacentes à massa primária da neoplasia ${ }^{1,3,4}$, o que é aceito como falha da TC. Além dos linfonodos tumorais, os linfonodos localizados no ligamento gastro-hepático ou na grande curvatura frequentemente não são detectados pela $\mathrm{TC}^{20}$. A dificuldade em diagnosticar linfadenopatia nessas regiões pode ser causada por metástases em linfonodos de tamanho normal, pela presença de estruturas vasculares múltiplas nessas regiões ou por ambas as situações, tornado esta detecção mais difícil ${ }^{7,26,27}$. Apesar da baixa sensibilidade dos achados da TC na detecção da linfadenopatia no CAG, os linfonodos com sinais de acometimento localizados pela TC no tronco celíaco ou na região para-aórtica podem não ser adequadamente verificados por procedimentos cirúrgicos e a presença de doença neste grupo de linfonodos é responsável por proporção significativa de insucesso de tratamento do câncer gástrico ${ }^{12,28,29}$. Entretanto, o tamanho médio dos linfonodos acometidos não costuma diferir significativamente do diâmetro dos linfonodos falso-positivos ${ }^{20}$. Os resultados falso-positivos que diminuem a sensibilidade podem ser resultado da hiperplasia reativa ou aumento benigno dos linfonodos devido à inflamação adjacente ou mesmo de infecção ${ }^{11,17,30,31}$. No presente estudo, não foram observados resultados falso-positivos da TC no tocante ao comprometimento linfonodal, provavelmente devido à baixa acuidade do exame na detecção de linfonodos.

Komaki et al..$^{32}$ relataram os resultados da comparação dos achados da TC abdominal com os achados histopatológicos e cirúrgicos em doentes com CAG. Esses autores verificaram que dois aspectos podem reduzir o número de falso-positivos na TC. Um deles é que, na TC, a linfadenopatia pode ser caracterizada como solitária ou em bloco, também denominada linfadenopatia múltipla. Esses autores citam que nesta última situação havia doença metastática em $96 \%$ dos doentes, ao passo que na linfadenopatia solitária, a doença metastática estava presente em apenas $48 \%$ dos casos. Assim, um linfonodo solitário aumentado é menos provável de ser metastático do que um grupo de linfonodos também aumentado. Outro aspecto que pode reduzir o número de falso-positivos na TC refere-se ao uso da TC dinâmica para intensificar a imagem dos linfonodos. O realce dos linfonodos normais pode alcançar $82 \%$, enquanto que habitualmente não se nota intensificação da imagem dos linfonodos comprometidos preenchidos por doença metastática. Portanto, o reconhecimento do realce de contraste em linfonodos reativos pode ajudar a diminuir o número de exames falso-positivos.
No presente estudo, empregamos a TC helicoidal, modelo do aparelho disponível por ocasião da realização dos exames. A TC multislice possui velocidade maior de escaneamento das imagens e permite uma colimação mais fina, o que pode promover a visibilização de neoplasias mais sutis, especialmente quando o agente de contraste é injetado intravenosamente em "bolus" e de maneira rápida. No caso do adenocarcinoma gástrico, embora essas vantagens da TC multislice possam ser atraentes, julgamos que, pelas dimensões avantajadas das lesões gástricas encontradas nas peças cirúrgicas, essas características não influenciaram os resultados do presente estudo.

O desenvolvimento da tecnologia de tomografia computadorizada multislice representou avanço tecnológico significativo na TC. O aumento da velocidade de imagem permite a digitalização com cortes mais finos, fornece maior flexibilidade na exploração radiológica durante as várias fases de realce e oferece a capacidade de realizar excelentes imagens tridimensionais ${ }^{15}$.

Considerando-se os linfonodos maiores que $1 \mathrm{~cm}$ no menor diâmetro como comprometidos por tumor, a sensibilidade da TC multislice é de mais de 95\%, mas a especificidade é de apenas 50\%. Com a utilização futura de contrastes superparamagnéticos linfotróficos à base de nanopartículas de ferro, a sensibilidade da TC multislice poderá atingir $100 \%$ e sua especificidade chegar a $95 \%$ no estadiamento linfonodal de neoplasias gastrintestinais ${ }^{17}$.

Em relação ao fígado, a TC multislice permite maior cobertura anatômica, com digitalização do fígado durante a fase venosa portal crítica, o que produz distinção máxima entre as lesões de baixa atenuação e do parênquima hepático normal, proporcionando maior acurácia para a maioria das metástases hepáticas ${ }^{20}$.

A quantidade de gordura perigástrica e a localização dos linfonodos são importantes fatores na determinação na sensibilidade da detecção de linfonodos $2,16,21$. A detecção de linfonodos pode ser dificultosa quando há pouca gordura, independentemente do tamanho e da localização anatômica do nódulo ${ }^{19,30}$. Também, linfonodos localizados num pequeno espaço e circundados por tecido mole, tal como ocorre na porta hepatis, podem ser difíceis de avaliar pelo mesmo motivo que a sensibilidade da TC é baixa para a detecção desses linfonodos nos doentes com pouca gordura perigástrica ${ }^{16,33}$. Uma limitação do estudo dos linfonodos com a TC em doentes com carcinoma gástrico se deve ao fato de que os linfonodos removidos não são mapeados um a um de maneira correspondente em relação aos achados na imagem ${ }^{34}$. $\mathrm{Na}$ presente série, tais circunstâncias podem ter contribuído para a baixa sensibilidade da TC na detecção de linfonodos infiltrados pelo CAG.

Existem muitos fatores, além do tamanho do linfonodo, que podem ajudar a explicar a diferença na sensibilidade para a detecção de linfonodos entre linfonodos comprometidos e não comprometidos por metástases ${ }^{35}$. A atenuação dos linfonodos na TC é importante porque radiologistas reconhecem os linfonodos pelo meio da diferença de atenuação entre linfonodos e a gordura ao seu redor ${ }^{34}$. Numa visibilização subjetiva da atenuação da TC de linfonodos, a atenuação elevada 
é frequente naqueles não comprometidos por metástases ${ }^{11,19,27,32}$. Outro possível fator é a atenuação da TC sem a utilização de material de contraste. É descrito que os linfonodos histopatologicamente negativos contêm mais gordura'. Assim, o realce do linfonodo na fase relativamente precoce após a ministração de material de contraste e a atenuação do nódulo na TC sem material de contraste podem também ter influenciado na baixa sensibilidade observada no presente estudo.

$\mathrm{Na}$ série aqui apresentada, foram considerados alterados os linfonodos que excederam $8 \mathrm{~mm}$ no maior diâmetro. A baixa acurácia para linfonodos pode ser creditada ao fato de que os linfonodos perigástricos são adjacentes ao tumor e não podem ser visto separadamente na $\mathrm{TC}^{35}$. Assim, a TC pode subestadiar linfonodos perigástricos especialmente quando eles estiverem próximos à massa neoplásica principal. Maior acurácia da TC é descrita no estadiamento de linfonodos perigástricos ou extragástricos ${ }^{31}$.

Outro fator que pode auxiliar a explicar a diferença na sensibilidade para a detecção de linfonodos com e sem metástases é a própria configuração de cada linfonodo. As imagens axiais da TC não são propensas a mostrar o maior diâmetro do linfonodo, e os linfonodos comprometidos tendem a ter comprimento diferente da sua largura?.

A TC pré-operatória é ainda indispensável na avaliação da presença de metástases distantes ${ }^{4,36,37}$. A exploração abdominal para o carcinoma gástrico não é realizada, primariamente, para o estadiamento da doença, mas sim na remoção da doença para possível cura ou paliação. Virtualmente, nenhum achado na TC deve afastar a laparotomia no doente com carcinoma gástrico ${ }^{38}$. No raro caso de os achados da TC sugerirem doença disseminada e quando não há obstrução gástrica ou evidência de sangramento gastrintestinal, os achados da TC podem evitar a laparotomia. Mesmo nesse caso, a confirmação da doença metastática por biópsia com agulha fina pode ser necessária, pois pode ocorrer exame de TC falso-positivo não apenas para linfadenopatia por comprometimento neoplásico, mas também para metástases peritoneais, hepáticas e invasão pancreática ${ }^{15,39}$. Nesse sentido, na presente casuística, os achados da TC não evitaram a laparotomia nos doentes desta série, pois, mesmo naqueles com metástases hepáticas reveladas pela
TC, havia indicação de procedimento cirúrgico por conta de obstrução pilórica e/ou hemorragia com origem na lesão gástrica. No tocante às metástases à distância, especialmente aquelas localizadas no fígado, os resultados obtidos nesta série com a TC são comparáveis com os resultados da maioria dos estudos ${ }^{4,10,20,21,40-42}$, embora outros autores ${ }^{18,25,43}$ tenham apontado sensibilidade de 51 a $97 \%$. Embora a incidência de falso-positivos não tenha ocorrido neste estudo, pode ser alta e provavelmente está relacionada com o fato de a interface entre o carcinoma gástrico e o lobo esquerdo do fígado ser frequentemente oblíqua ao plano de escaneamento ${ }^{31}$.

Assim como o presente estudo, outras séries ${ }^{6,7,10,42}$ falharam em detectar carcinomatose peritoneal.

A TC pode modificar a abordagem cirúrgica, sugerindo doença irressecável ou avançada ${ }^{4,10,11,19,30}$. A baixa percentagem de doentes hiperestadiados pela TC, combinada com a acurácia da TC helicoidal abdominal na doença avançada quando comparada com os achados da laparotomia, apoia seu uso pré-operatório. Dessa maneira, a TC raramente hiperestima o estadiamento da doença e raramente previne a exploração de doentes que são potencialmente curáveis ${ }^{2}$, como ocorreu nos doentes do presente estudo cujo resultado da TC não motivou a suspensão da laparotomia.

Autores $2,8,11,16,34$ sugeriram que a exploração operatória deve ser realizada independentemente dos resultados da TC, e a laparotomia deve ser considerada padrão ouro no estadiamento do CAG. Entretan-

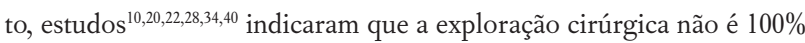
acurada, especialmente no tocante à presença não revelada de invasão pancreática, metástases hepáticas e de comprometimento linfonodal pela neoplasia.

Nas condições deste estudo, os resultados obtidos permitiram concluir que o exame de tomografia computadorizada helicoidal abdominal realizado no pré-operatório de doentes com carcinoma gástrico apresentou sensibilidade elevada na detecção de metástases hepáticas e sensibilidade baixa na detecção do comprometimento linfonodal pela neoplasia, além de não ter sido capaz de identificar metástases peritoneais.

\section{Referências}

1. Prado FC, Ramos JA, Valle JR. Atualização Terapêutica: Manual Prático de Diagnostico e Tratamento. In: Borges DR, Rothschild HA, editores. Câncer de Estômago. 22a ed. São Paulo: Artes Médicas; 2005. p. 566-9.

2. Paramo JC, Gomez G. Dynamic CT in the preoperative evaluation of patients with gastric cancer: correlation with surgical findings and pathology. Ann Surg Oncol. 1999;6(4):379-84.

3. Brancato $S$, Winner TJ. Surgical management of gastric cancer: review and consideration for total care of the gastric cancer patient. Curr Treat Options Gastroenterol. 2008:11(2):109-18.
4. Habermann CR, Weiss F, Riecken R, Honarpisheh H, Bohnacker S, Staedtler $\mathrm{C}$, et al. Preoperative staging of gastric adenocarcinoma: comparison of helical CT and endoscopic US. Radiology. 2004;230(2): $465-71$

5. Incidência de Câncer no Brasil: estimativa 2008: síntese de resultados e comentários. [citado 5 mai 2008]. Disponível em: www.inca.gov.br/ estimativa/2008/index.asp?link= conteudo view.asp\&lD $=5$.

6. Tschmelitsch J, Weiser MR, Karpeh MS. Modern staging in gastric cancer. Surg Oncol. 2000;9(1):23-30. 
7. Wakelin SJ, Deans C, Crofts TJ, Allan PL, Plevris JN, Paterson-Brown S. A Comparison of computerized tomography, laparoscopic ultrasound and endoscopic ultrasound in the preoperative staging of esophago-gastric carcinoma. Eur J Radiol. 2002;41(2):161-7.

8. Ziegler K, Sanft C, Zimmer T, Zeitz M, Felsenber D, Stein H, et al. Comparison of computerized tomography, endosonography and intraoperative assessment in TN staging of gastric carcinoma. Gut. 1993;34(5):604-10.

9. Fukuya T, Honda H, Hayashi T, Kaneko K, Tateshi Y, Ro T, et al. Lymph-node metastases : efficacy for detection with helical CT in patients with gastric cancer. Radiology. 1995;197(3):705-11.

10. Davies J, Chalmers AG, Sue-Ling HM, May J, Miller GV, Martin IG, et al. Spiral computed tomography and operative staging of gastric carcinoma: a comparison with histopathological staging. Gut. 1997;41(3):314-9

11. Kumano $S$, Murakami $T$, Kim $T$, Hori $M$, lannaccone $R$, Nakata $S$, et al. T staging of gastric cancer: role of multi-detector row CT. RSNA. 2005;237(3):961-6.

12. Barry JD, Edwards P, Lewis WG, Dhariwal D, Thomas GV. Special interest radiology improves the perceived preoperative stage of gastric cancer. Clin Radiol. 2002;57(11):982-3.

13. Sarela Al, Lefkowitz R, Brennan MF, Karpeh MS. Selection of patients with gastric adenocarcinoma for laparoscopic staging. Am J Surg. 2006;191(1):134-8.

14. Triantafillidis JK, Cheracakis P. Diagnostic evaluation of patients with early gastric cancer - a literature review. Hepato-gastroenterol. 2004;51:618-24.

15. Chamadol N, Wongwiwatchai J, Bhudhisawasd V, Pairojkul C. Accuracy of spiral CT in preoperative staging of gastric carcinoma: correlation with surgical and pathological findings. J Med Assoc Thai. 2008;91(3):356-63.

16. Lee DH, Seo TS, Ko YT. Spiral CT of the gastric carcinoma: staging and enhancement pattern. J Clin Imag. 2001;25(1):32-7.

17. Wei WZ, Yu JP, Li J, Liu CS, Zheng XH. Evaluation of contrast-enhanced helical hydro-CT in staging gastric cancer. World $\mathrm{J}$ Gastroenterol. 2005;11(29):4592-5.

18. Botet JF, Lightdale CJ, Zauber AG, Gerder H, Winawer SJ, Urmacher C, et al. Preoperative staging of gastric cancer: comparison of endoscopic US and dynamic CT. Radiology. 1991;181(2):426-32.

19. Blackshaw GR, Stephens MR, Lewis WG, Boyce J, Barry JD, Edwards P, et al. Progressive CT system technology and experience improve the perceived preoperative stage of gastric cancer. Gastric Cancer. 2005;8(1):29-34.

20. Wei WZ, Yu JP, Li J, Liu CS, Zheng XH. Evaluation of contrast-enhanced helical hydro-CT in staging gastric cancer. World $\mathrm{J}$ Gastroenterol. 2005;11(29):4592-5.

21. Ianora AA, Pedote P, Scardapane A, Memeo M, Rotondo A, Angelelli G. Preoperative staging of gastric carcinoma with multidetector spiral CT. Radiol Med (Torino). 2003;106(5-6):467-80.

22. Madden MV, Price SK, Learmonth GM, Dent DM. Surgical staging of gastric carcinoma: sources and consequences of errors. Br J Surg. 1987;74(2): 19-21.
23. Kleinhaus U, Militianu D. Computed tomography in the preoperative evaluation of gastric carcinoma. Gastrointest Radiol. 1988;13(2):97-101.

24. Dehn TC, Reznek RH, Nockler IB, White FE. The preoperative assesment of advanced gastric cancer by computed tomography. $\mathrm{Br} \mathrm{J}$ Surg. 1984;71(6):413-7.

25. Moss AA, Schnyder P, Marks W, Margulis AR. Gastric adenocarcinoma: a comparison of the accuracy and economics of staging by computed tomography and surgery. Gastroenterology. 1981;80(1):45-50.

26. Chen F, Ni Y, Zheng K, Ju S, Sun J, Ou X, et al. Spiral CT in gastric carcinoma: comparison with barium study, fiberoptic gastroscopy and histopathology. World J Gastroenterol. 2003;9(7):1404-8.

27. PolkowskiM,PaluckiJ, WronskaE, Szawlowski A, Nasierowska-Guttmejer A, Butruk E. Endosonography versus helical computed tomography for locoregional staging of gastric cancer. Endoscopy. 2004;36(7): 617-23.

28. Kadowaki K, Murakami T, Yoshioka H, Kim T, Takahashi S, Tomoda K, et al. Helical CT imaging of gastric cancer: normal wall appearance and the potential for staging. Radiation Med. 2000;18(1):47-54.

29. Lee DH. Two-dimensional and three-dimensional imaging of gastric tumors using spiral CT. Abdom Imaging. 2000;25(1):1-6.

30. Horton KM, Fishman EK. Current role of CT in imaging of the stomach. Radiographics. 2003;23(1):75-87

31. Mani NBS, Suri S, Gupta S, Wig JD. Two-phase dynamic contrast-enhanced computed tomography with water-filling method for staging of gastric carcinoma. J Clin Imaging. 2001;25(1):38-43.

32. Komaki S, Toyoshima S. CT's capability in detecting advanced gastric cancer. Gastrointest Radiol. 1983:8:307-13.

33. Sussman SK, Halvorsen RA, Illescas FF, Cohan RH, Saeed M, Silverman PM, et al. Gastric adenocarcinoma: CT versus surgical staging. Radiology. 1988;167(2):335-40

34. Giner RG, Sos JE, Sanchís JL, del Castillo JR, Vila JH, Valdivieso EM. Helical $\mathrm{CT}$ evaluation in the preoperative staging of gastric adenocarcinoma. Rev Esp Enferm Dig. 2002:94(10):593-600.

35. Balfe DM, Koehgler RE, Karstaedt M, Stanley RJ, Sagel SS. Computed tomography of gastric neoplasms. Radiology. 1981;140:431-6.

36. Fusaroli P, Caletti G. Endoscopic ultrasonography: current clinical role. Eur J Gastroenterol Hepatol. 2005;17(3):293-301.

37. Xi WD, Zhao C, Ren GS. Endoscopic ultrasonography in preoperative staging of gastric cancer: determination of tumor invasion depth, nodal involvement and surgical resectability. World J Gastroenterol. 2003;9(2):254-7.

38. Feussner H, Omote K, Fink U, Walker SJ, Siewert JR. Pretherapeutic laparoscopic staging in advanced gastric carcinoma. Endoscopy. 1999;31(5):342-7.

39. Meining A, Rosch T, Wolf A, Lorenz R, Allescher HD, Kauer W, et al. High interobserver variability in endosonography staging of upper gastrointestinal cancers. Z Gastroenterol. 2003;41(5):391-4. 
40. Ba-Ssalamah, Prokop M, Uffmann M, Pokieser P, Teleky B, Lechner G. Dedicated multidetector CT of the stomach: spectrum of diseases. Radiographics. 2003;23(3):625-44.

41. Sohn KM, Lee JM, Lee SY, Ahn BY, Park SM, Kim KM. Comparing MR imaging and CT in the staging of gastric carcinoma. AJR. 2000;174(6): 1551-7.
42. Giner GR, Sos JE, Sanchis JLS, Castillo JR, Vila JHG, Valdivieso EM. Helical CT evaluation in the preoperative staging of gastric adenocarcinoma. Rev Esp Enferm Dig. 2002;94(10):593-600.

43. Cook AO, Levine BA, Sirinek KR, Gaskill HV. Evaluation of gastric adenocarcinoma. Abdominal computed tomography does not replace celiotomy. Arch Surg. 1986;121(5):603-6. 